\title{
Experiments with crystal deflectors for high energy ion beams: Electromagnetic dissociation probability for well channeled ions
}

\author{
W. Scandale, ${ }^{1,2,3}$ A. M. Taratin, ${ }^{4}$ and A. D. Kovalenko ${ }^{4}$ \\ ${ }^{1}$ CERN, European Organization for Nuclear Research, CH-1211 Geneva 23, Switzerland \\ ${ }^{2}$ Laboratoire de l'Accelerateur Lineaire (LAL), Universite Paris Sud Orsay, Orsay, France \\ ${ }^{3}$ INFN Sezione di Ferrara, Dipartimento di Fisica, Università di Ferrara, Ferrara, Italy \\ ${ }^{4}$ Joint Institute for Nuclear Research, 141980, Dubna, Moscow Region, Russia
}

(Received 30 September 2012; published 3 January 2013)

\begin{abstract}
The paper presents the current status with the use of the crystal defectors for high energy ion beams. The channeling properties of multicharged ions are discussed. The results of the experiments on the deflection and extraction (collimation) of high energy ion beams with bent crystals performed in the accelerator centers are shortly considered. The analysis of the recent collimation experiment with a $\mathrm{Pb}$ nuclei of $270 \mathrm{GeV} / c$ per charge at the CERN Super Proton Synchrotron showed that the channeling efficiency was as large as about $90 \%$. For $\mathrm{Pb}$ ions of the LHC energies a new mechanism, which can reduce the channeling efficiency, appears. The electromagnetic dissociation (ED) becomes possible for well channeled particles. However, the estimations performed in the paper show that the ED probability is small and should not visibly reduce the collimation efficiency. On the other hand, the aligned crystal gives the possibility to study the ED processes of heavy nuclei in the conditions when nuclear interactions are fully suppressed.
\end{abstract}

DOI: 10.1103/PhysRevSTAB.16.011001

\section{INTRODUCTION}

When a high-energy charged particle enters the crystal with a small angle relative to some crystallographic planes, its motion is governed by the crystal potential averaged along the planes [1]. If the angle is smaller than the critical channeling angle,

$$
\theta_{c o}=\left(2 U_{o} / p v\right)^{1 / 2},
$$

where $p$ and $v$ are the particle momentum and velocity, and $U_{o}$ is the depth of the planar potential well, the particle can be captured into the planar channeling regime oscillating between two neighboring crystal planes. The averaged electric field of the crystal planes reaches tremendous values. So, its maximal strength $E_{m}$ along the (110) planes in silicon crystals is about $6 \mathrm{GV} / \mathrm{cm}$. Particle channeling is still possible if the crystal is bent with a radius larger than the critical one, $R>R_{c}$ [2],

$$
R_{c}=\frac{p v}{Z_{1} e E_{m}},
$$

where $Z_{1} e$ is the particle charge. Thus, channeled particles should be deflected by the bend angle of the crystal.

The possibility of charged particle deflection by bent crystals in the channeling regime had been shown in the experiment performed with $8.9 \mathrm{GeV}$ protons at the external beam of the Joint Institute for Nuclear Research (JINR) synchrophasotron [3]. Afterwards, particle channeling in

Published by the American Physical Society under the terms of the Creative Commons Attribution 3.0 License. Further distribution of this work must maintain attribution to the author(s) and the published article's title, journal citation, and DOI.
PACS numbers: 29.27. $-\mathrm{a}, 61.85 .+\mathrm{p}$

bent crystals had been studied in many experiments at the CERN, Institute for High Energy Physics (IHEP), and Fermi National Accelerator Laboratory (see the reviews [4-6]). Bent crystals are successfully used at the IHEP to extract the proton beam from the synchrotron U70 and for the beam splitting between the experiments [7]. At the present time, the possibility to use a crystal deflector as a primary collimator of the LHC beam halo collimation system is studied in the UA9 experiment at the Super Proton Synchrotron (SPS) beams [8-10]. Halo particles of the SPS circulating beam are deflected by the crystal and directed onto the secondary collimator absorber. In contrast to the amorphous primary collimator, the crystal one directs particles far from the absorber edge decreasing probability of their scattering back to the circulating beam.

The experiments performed in JINR, CERN, and Brookhaven National Laboratory [11-21] showed that multicharged ions are also successfully deflected by bent crystals. However, electromagnetic dissociation (ED) becomes possible for heavy nuclei of the LHC energies even for well channeled particles, which should be taken into account.

In this paper, the peculiarities of channeling for multicharged ions (heavy nuclei) in bent crystals and the results of the experimental studies on the ion deflection are shortly considered. The probability estimation of electromagnetic dissociation for $\mathrm{Pb}$ ions of the LHC energies at channeling along the (110) planes in silicon crystals is given.

\section{CHANNELING PECULIARITIES OF MULTICHARGED IONS}

The force acting on a charged particle in the averaged electric field of the crystal planes as well as the potential 
well depth of planar channels are increased proportionally to the particle charge $Z_{1}$. The critical transverse energy of particles for channeling increases accordingly. The critical channeling angle for relativistic particles is determined by the ratio $p_{z}=p / Z_{1}$, therefore its value is the same for ions with the same value of $p_{z}$. The other channeling characteristics determined by the averaged electric field of planar channels like the spatial period of oscillations in the channels $\lambda$ and the probability of capture into the channeling regime $P_{c}$ are also invariant with respect to $p_{z}$.

Particles leave a channeling regime because of scattering by the crystal electrons and nuclei. The transverse momentum acquired by a particle due to scattering is proportional to its charge, whereas the scattering angle $\theta \sim Z_{1} / p$. Thus, the dechanneling length of ions is also invariant with respect to $p_{z}$.

The bend radius value of the crystal planes, which makes channeling impossible, the critical radius $R_{c}$ (2) is also invariant with $p_{z}$. In the parabolic approximation for the planar channel potential, the critical angle dependence on the crystal radius $R$ is described as

$$
\theta_{c}\left(R, p_{z}\right)=\theta_{c o}\left(p_{z}\right)\left[1-R_{c}\left(p_{z}\right) / R\right] .
$$

Therefore, the critical angle and the other parameters of channeling and dechanneling in a bent crystal are also invariant with $p_{z}$. This leads to the invariance of the deflection efficiency of particles by a bent crystal with respect to $p_{z}$.

However, the cross section of nuclear interactions and ionization energy losses significantly increase for heavy nuclei. This practically does not change the deflection efficiency of the nuclei beam at its single passage through the crystal. A visible difference appears only when a bent crystal is used for the extraction or collimation of the accelerator beam. The increasing losses of heavy nuclei in the crystal due to nuclear interactions reduce the possibility of their multiple passages through the crystal. Besides, large ionization energy losses of heavy nuclei in the crystal, which are proportional to $Z_{1}{ }^{2}$, lead to large reductions of particle momenta. Their relative deviations from the synchronous momentum $p_{o}, \delta=p / p_{o}-1$, increase. Thus, the orbits of heavy ions after the crystal passage acquire considerable shifts, which are equivalent to the changes of their oscillation amplitudes.

\section{EXPERIMENTS WITH BENT CRYSTALS}

The first experiment on the deflection of nuclei by a bent crystal had been performed at the JINR synchrophasotron [11]. The beam of carbon nuclei with the total momentum of $53 \mathrm{GeV} / c$ was deflected by $65 \mathrm{mrad}$ using the $2 \mathrm{~cm}$ silicon crystal bent along the (111) planes. The suggestion of using the crystal deflector to extract the nuclear beam from the relativistic ion collider like RHIC and LHC and the estimation of the extraction efficiency for $100 \mathrm{GeV} / u$ $\mathrm{Au}$ nuclei were given in [12]. Later, the scheme of the nuclear beam extraction from the superconducting synchrotron nuclotron in JINR using a bent crystal of silicon [13] and tungsten [14] had been proposed and studied by simulation. The experiments on the beam extraction from the JINR nuclotron [15] and deflection of the extracted beam by bent silicon crystals have been performed for deuterons with the energy of $2-3 \mathrm{GeV} / u$ [16]. The crystal bend angles along the (111) planes were 100 and $56 \mathrm{mrad}$, respectively.

The experiments on the nuclei deflection by a bent crystal at the CERN SPS synchrotron have been performed mainly with $\mathrm{Pb}$ nuclei [17-20]. In the experiments, the $60 \mathrm{~mm}$ long silicon crystal bent along the (110) planes with a three-point bending device was used. The crystal length was comparable with the dechanneling length for the well channeled fraction, which is determined by multiple scattering on the crystal electrons and equals about $20 \mathrm{~cm}$ for particles with $p_{z}=400 \mathrm{GeV} / c$. The observed deflection efficiency for the $\mathrm{Pb}$ nuclei beam with $p_{z}=400 \mathrm{GeV} / c$ was $9 \%$ and $13 \%$ for the crystal bent by the angles of 8.9 and $4.3 \mathrm{mrad}$, respectively. The simulation of the experiments performed according to the model described in the review [5] gave the results, which agree with the experiment with the accuracy better than $15 \%$. The comparison of the deflection efficiencies obtained in the experiment and in the simulation is presented in Table 1 in Ref. [18]. The particle trajectories were calculated in the averaged potential of bent crystal planes. The change of the particle transverse velocity due to multiple scattering by the crystal electrons and nuclei was performed in steps along the trajectory, which were much smaller than the spatial period of particle oscillations in the planar channel, taking into account the realistic distributions of electrons and nuclei in the channels.

In the experiment at the CERN SPS [19], the scintillation detector installed at some transverse distance from the straight and deflected beams was used to measure the emission of charged particles from the crystal induced by the passage of $\mathrm{Pb}$ nuclei through the crystal. It was shown that the $\mathrm{Pb}$ nuclei losses due to nuclear interactions were reduced for the aligned crystal by a value approximately equal to the deflected beam fraction.

In the experiment at the CERN SPS [20], the charge states of the beam of $\operatorname{In}^{49+}$ nuclei with $370 \mathrm{GeV} / c$ per charge deflected by the silicon crystal bent by the angles from $7.5 \mathrm{mrad}$ up to $19.8 \mathrm{mrad}$ were studied. The accelerated nuclei passing through the crystal may lose a few protons due to nuclear interactions. The charge of particles left the crystal was measured by the ionization chamber. It was shown that the fragmentation probability of nuclei in the deflected beam was more than 20 times smaller than for the straight beam passed through the nonaligned crystal.

The experiment on the extraction of $\mathrm{Pb}$ nuclei with $270 \mathrm{GeV} / c$ per charge from the CERN SPS has been made using $4 \mathrm{~cm}$ long silicon crystal bent along the 
(110) planes by the angle $8.5 \mathrm{mrad}$ [17]. The extraction efficiency was about $10 \%$, which is about 2 times smaller than for the extraction of the SPS proton beam with the same crystal. The width of the orientational dependence of the extraction efficiency being about $50 \mu \mathrm{rad}$ was also considerably smaller than for the extraction of protons. Both these circumstances were caused by the reduction of the contribution of multiple passages of nuclei through the crystal because of the increase of losses due to nuclear interactions for particles uncaptured into the channeling regime.

The use of bent crystals for the collider beam collimation is very promising. In this case, a few millimeters long crystal can provide a small deflection angle of the order of $100 \mu \mathrm{rad}$ required to direct particles on the absorber. In such short crystals, only particles with large oscillation amplitudes in the planar channels can be lost from the channeling regime mainly because of Coulomb scattering by the crystal nuclei.

The first experiment on the nuclei collimation has been realized at the collider RHIC for the beams of Au nuclei with $p_{z}=250 \mathrm{GeV} / c$ [21]. The $5 \mathrm{~mm}$ long silicon crystal bent along the (110) planes by the angle $440 \mu \mathrm{rad}$ was used. The yield of secondary particles generated by the beam halo nuclei in the crystal was measured by the beam loss monitors downstream the crystal. The beam losses in the crystal were reduced by $25 \%$ for the crystal orientation provided the deflection of channeled nuclei.

In the UA9 experiment on the study of the crystal collimation at the CERN SPS, one of the $2 \mathrm{~mm}$ long silicon crystals with the bend angle about $170 \mu \mathrm{rad}$ was used as a primary collimator. The studies were performed both with protons and $\mathrm{Pb}$ nuclei with $p_{z}=120 \mathrm{GeV} / c$ and $270 \mathrm{GeV} / c[9,10]$. Figure 1 shows the dependence of the $\mathrm{Pb}$ nuclei losses in the crystal on its orientation observed in the experiment [10]. The losses were reduced more than 7 times in the aligned case (a deep minimum near zero). The simulation results (curve 2) well describe this reduction of inelastic interactions of $\mathrm{Pb}$ ions in the aligned crystal. The simulation shows that the reduction value can be provided only when about $90 \%$ of particles hitting the crystal are deflected in the channeling regime by the bend angle (channeling efficiency).

The reduction of inelastic interactions of ions in the aligned crystal can be estimated using a simple consideration. For nuclear interactions, the impact parameter of nuclei collisions should be smaller than the sum of the nuclei radii, $R_{\mathrm{in}}=R_{\mathrm{Si}}+R_{\mathrm{Pb}} \approx 10 \mathrm{fm}$, which is much smaller than a planar channel width in a crystal. Figure 2 shows the potential of the (110) planar channel in a silicon crystal for $\mathrm{Pb}$ nuclei (solid curve), the channel width $d_{p}=$ $1.92 \AA$. The vertical dot-dashed lines show the regions (nuclear corridors) where the crystal nuclei are located near the channel walls (the edge points of the figure). The distribution of the crystal nuclei caused by the thermal

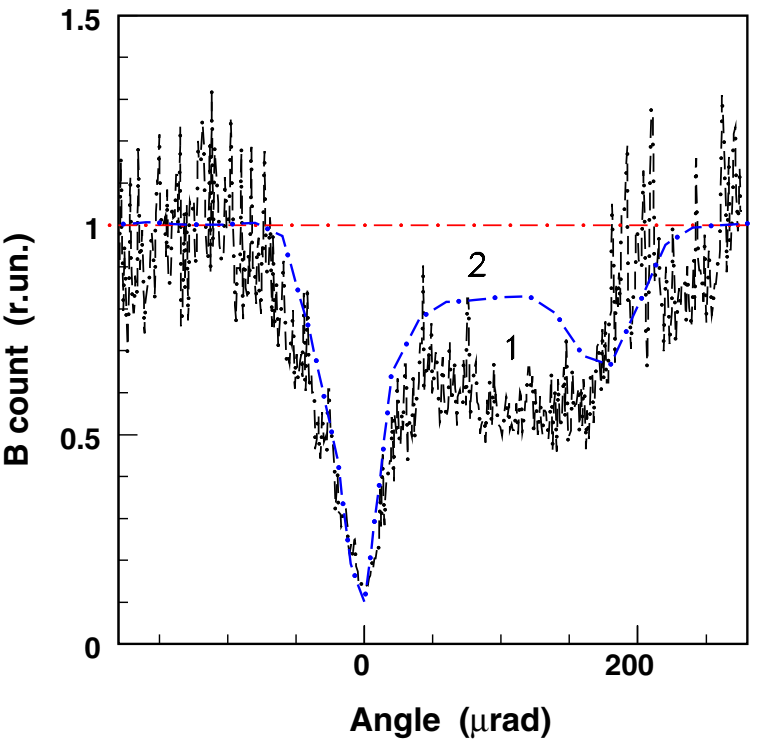

FIG. 1. The UA9 experiment results on the crystal collimation of the CERN SPS beam of $\mathrm{Pb}$ ions with $270 \mathrm{GeV} / c$ per charge, the reproduction of Fig. 5a from Ref. [10]. (1) The experimental dependence of beam losses in the crystal on its angular position normalized to the value for the amorphous orientation of the crystal (dot-dashed line). (2) The dependence of the number of inelastic nuclear interactions of nuclei in the crystal on its orientation angle obtained by simulation. The beam loss reduction at the channeling condition (near zero) is larger than 7.

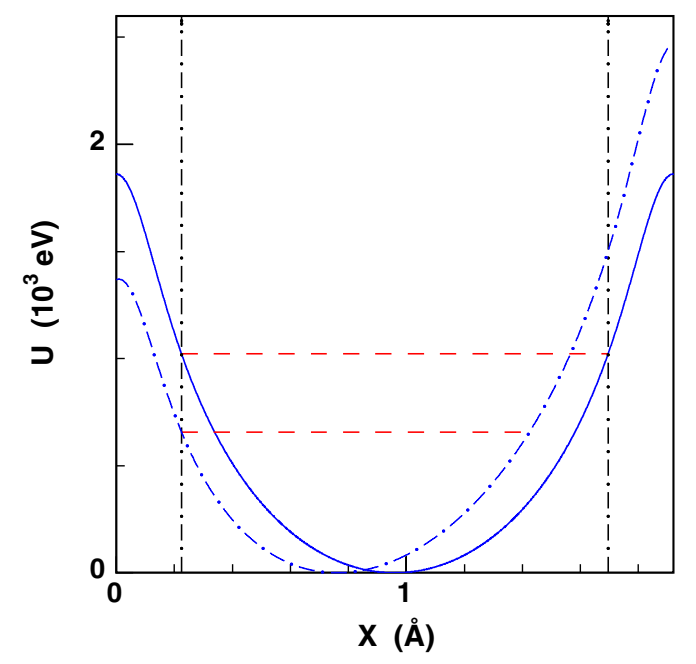

FIG. 2. The potential of the (110) planar channel in an unbent silicon crystal for $\mathrm{Pb}$ nuclei (solid curve) and the effective potential of the channel bent with $R=100 \mathrm{~m}$ for $\mathrm{Pb}$ nuclei with $p_{z}=7 \mathrm{TeV} / c$ (dot-dashed curve). The vertical dot-dashed lines show the regions near the channel walls where the crystal nuclei are located (nuclear corridors). The horizontal dashed lines show the channel part where the trajectories of well channeled particles are situated. Well channeled ions do not approach to the channel walls. Thus, nuclear interactions for them are forbidden and ED events are strongly suppressed. 
TABLE I. Data on the crystal extraction (collimation) of heavy nuclei.

\begin{tabular}{lccccc}
\hline \hline $\begin{array}{l}\text { Nuclei, } \\
p_{z}(\mathrm{GeV} / c)\end{array}$ & $\begin{array}{c}\text { Crystal } \\
\text { length }(\mathrm{cm})\end{array}$ & $\begin{array}{c}\text { Bend } \\
\text { angle }(\mathrm{mrad})\end{array}$ & $\begin{array}{c}\text { Loss } \\
\text { reduction }\end{array}$ & $\begin{array}{c}\text { Channeling } \\
\text { efficiency, \% }\end{array}$ & $\begin{array}{c}\text { Accelerator, } \\
\text { date, reference }\end{array}$ \\
\hline $\mathrm{Pb}, 270$ & 4.0 & 8.5 & $\ldots$ & 10 & SPS, 1997, [17] \\
$\mathrm{Au}, 250$ & 0.5 & 0.44 & 0.25 & $\ldots$ & RHIC, 2005, [21] \\
$\mathrm{Pb}, 270$ & 0.2 & 0.17 & 7 & 90 & SPS, 2012, [10] \\
\hline \hline
\end{tabular}

vibrations has a Gaussian shape with $\sigma=u_{1}$, where $u_{1}$ is the thermal vibration amplitude, in the silicon crystals at the room temperature $u_{1}=0.075 \AA$. For the width of the nuclear corridor shown in Fig. 2, the value of $3 u_{1}$ was accepted, it is about $20 \%$ of the channel width. Well channeled particles with the whole trajectories situated in the central part of the planar channels outside the nuclear corridors cannot have nuclear interactions with the crystal nuclei. Thus, the reduction of particle losses in a single passage of a parallel beam through the aligned crystal can reach the value of about 5 . For the beam halo particles in the collimation experiment, which were not deflected due to channeling and avoided nuclear interactions at the first passage through the crystal, there is the possibility to be deflected in the following passages. This caused the additional increase of the beam loss reduction observed in the experiment [10].

On the right of the minimum there is the angular region with reduced losses caused by the volume reflection of particles in the crystal. The angular kicks of particles due to volume reflections are larger than the kicks due to Coulomb multiple scattering. Therefore, in volume reflection mode the particles perform a smaller number of passages through the crystal to reach the absorber aperture than for the crystal "amorphous" orientations far from the plane. This reduces the total number of inelastic interactions in the crystal.

The beam loss monitors installed in the first high dispersion area behind the absorber showed the same reduction of the off-momentum beam fraction, which forms the collimation leakage. It is clear because the off-momentum particles with large ionization energy losses as well as the beam losses in the crystal are generated by the same halo fraction uncaptured into the channeling regime. It should be noted that the studies of the crystal radiation resistance are required to resolve a question about possibility to use the crystal primary collimator for the heavy nuclei beams.

Table I summarizes the data on the extraction (collimation) of high energy nuclei beams mentioned above. Here the loss reduction is the ratio of particle losses for the amorphous and channeling orientations of the (110) silicon crystals.

\section{ED PROBABILITY FOR CHANNELED NUCLEI}

The cross section of inelastic nuclear interactions for $\mathrm{Pb}$ nuclei with $270 \mathrm{GeV} / c$ per charge in silicon crystal is $\sigma_{h}=4.3 \mathrm{~b}$ [10], which is about 10 times larger than for protons. Besides, already for this energy of $\mathrm{Pb}$ nuclei the ED cross section is comparable with the nuclear one, $\sigma_{\text {ed }}=1.37 \mathrm{~b}$. Thus, the attenuation length for $\mathrm{Pb}$ nuclei in the crystal primary collimator nonaligned with the beam in the experiment [10] was about $3.5 \mathrm{~cm}$.

Nuclear interactions can occur in the aligned crystal only for channeled particles with large oscillation amplitudes in the channels, which enter the nuclear corridors. In this case the probability of nuclear interactions still may be calculated using the nuclear cross section as $P_{h}=n \sigma_{h} L$, where $n$ is the atomic density and $L$ is the crystal length. However, the density $n$ should be averaged along the trajectories and ensemble of the beam particles.

The cross-section conception assumes a uniform distribution of the impact parameters of incident particles with the target nuclei. For nuclear interactions in the aligned crystal, the impact parameter distribution is close to uniform for very small parameters involved. However, the ED processes of high energy ions in the aligned crystals are possible for sufficiently large impact parameters comparable with the channel width (see below). Therefore, the assumption about the uniformity of the impact parameter distribution is not already valid and the cross-section conception for the ED processes of high energy ions in the aligned crystals is not applicable. In this case the ED probability should be straight calculated. In [22], the ED probability of deuterons with energy up to $5 \mathrm{GeV}$ in the aligned tungsten crystal was determined along the real calculated trajectories using the equivalent photon method.

In the coordinate system connected with an incident particle, the charge of the target nuclei provides a short electromagnetic pulse, which can be presented by the virtual photon flow. The minimal energy of photons exciting the giant dipole resonance of $\mathrm{Pb}$ nuclei with the emission of one neutron is $E_{n 1}=7.4 \mathrm{MeV}$. A maximal energy of the virtual photons in the collisions with the impact parameter $b$ can be estimated according to [23] as

$$
E_{\gamma m}=\gamma \hbar c / b
$$

where $\gamma$ is the relativistic factor of particles. $E_{\gamma m}$ reaches the value of $E_{n 1}$ for $\mathrm{Pb}$ nuclei with $p_{z}=270 \mathrm{GeV} / c$ $(\gamma=114.3)$ at $b=0.03 \AA$. Thus, in this case the electromagnetic dissociation can occur only for a small fraction of 
well channeled nuclei with trajectories approaching closely to the nuclear corridors. On the contrary, at the LHC energies for $\mathrm{Pb}$ nuclei with $p_{z}=7 \mathrm{TeV} / c(\gamma=$ 2963), the ED is possible even for channeled particles moving along the center of the (110) channels in silicon crystals when the impact parameter $b=d_{p} / 2=0.96 \AA$.

The ED probability in the nuclei collision with the impact parameter $b$ is calculated using the equivalent photon approach as [23]

$$
P(b)=\int_{E_{n 1}}^{\infty} N\left(E_{\gamma}, b\right) \sigma\left(E_{\gamma}\right) \frac{d E_{\gamma}}{E_{\gamma}}
$$

where $N\left(E_{\gamma}, b\right)$ is the number of the equivalent photons incident on the target per unit area, and $\sigma\left(E_{\gamma}\right)$ is the photodissociation cross section of $\mathrm{Pb}$ nuclei. The expression, taking into account the screening of target nuclei by atomic electrons [24], was used for the equivalent photon number. The experimental data for the photodissociation cross section of $\mathrm{Pb}$ nuclei were used. Figure 3(a) shows the calculated dependence of the ED probability of $\mathrm{Pb}$ nuclei due to collisions with $\mathrm{Si}$ nuclei per $1 \mathrm{~mm}$ path on the momentum $p_{z}$ for the idealized case of the particle motion along the center of the (110) silicon channel when the impact parameter to the channel walls $x_{o}=d_{p} / 2=$ $0.96 \AA\left(3.1 \times 10^{6} \mathrm{Si}\right.$ nuclei per $1 \mathrm{~mm}$ are on the right and on the left side).

The ED probability for the given impact parameter (5) should be averaged by the thermal vibrations of the crystal atoms $\left(x_{a}, y_{a}\right)$ and by the vertical coordinates of the particle entrance points $y_{o}$,

$$
\begin{aligned}
\bar{P}(x)= & \frac{1}{2 \pi u_{1}} \frac{2}{d} \int_{0}^{d / 2} d y_{o} \int_{-3 u_{1}}^{3 u_{1}} d y_{a} \\
& \times \int_{-3 u_{1}}^{3 u_{1}} P\left(\sqrt{\left(x-x_{a}\right)^{2}+\left(y_{o}-y_{a}\right)^{2}}\right) \\
& \times \exp \left(-\frac{x_{a}^{2}+y_{a}^{2}}{2 u_{1}^{2}}\right) d x_{a},
\end{aligned}
$$

as well as along the channeled particle trajectory with the impact parameter to the crystal plane $x_{o}$ in the channel potential $u(x)$,

$\tilde{P}\left(x_{o}\right)=\int_{0}^{d_{p} / 2} \frac{\bar{P}(x) d x}{\sqrt{u\left(x_{o}\right)-u(x)}} / \int_{0}^{d_{p} / 2} \frac{d x}{\sqrt{u\left(x_{o}\right)-u(x)}}$.

Figure 3(b) shows the calculated dependence of the ED probability of $\mathrm{Pb}$ nuclei with $p_{z}=7 \mathrm{TeV} / c$ averaged according to (6) and (7) on the impact parameter $x_{o}$ to the (110) planes of a silicon crystal in the case of parallel incidence. The ED probability per $1 \mathrm{~mm}$ is larger than $10^{-4}$ for particles entering the crystal closely to the nuclear corridor when $x_{o}$ is close to $3 u_{1}$ value. The ED probability
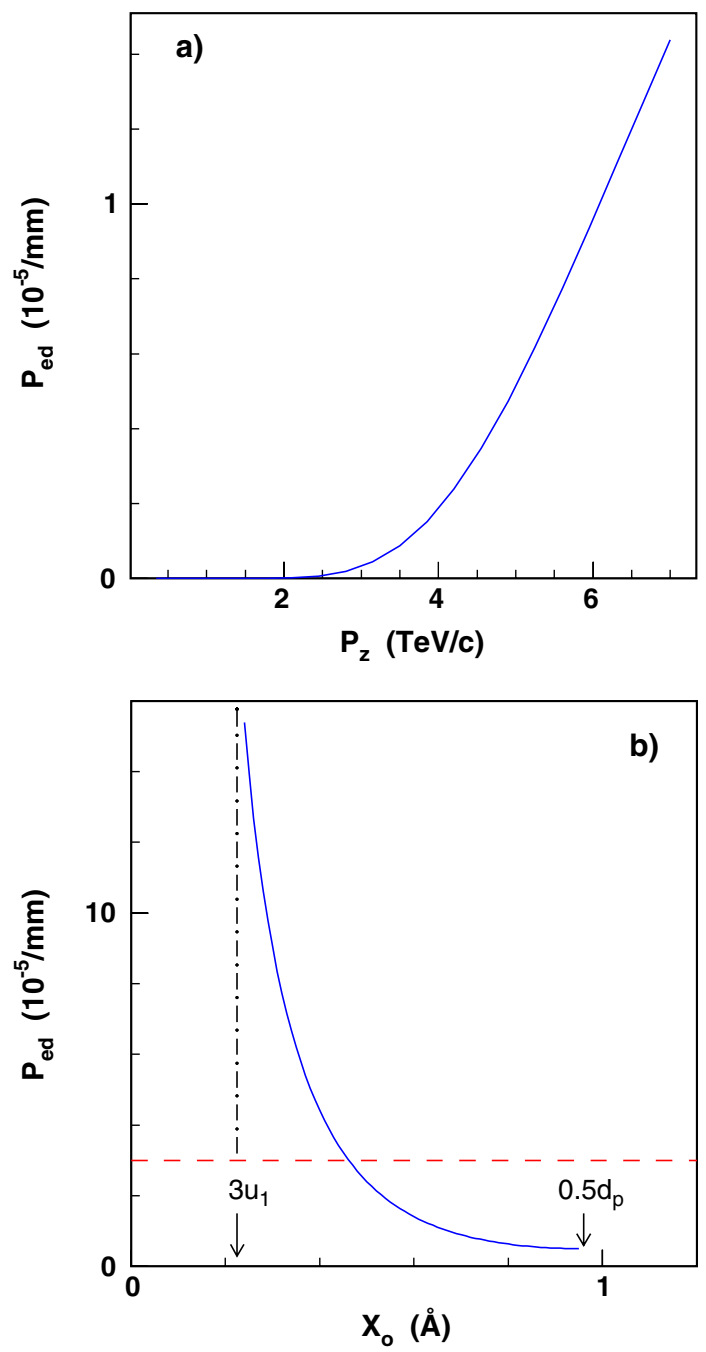

FIG. 3. (a) The calculated dependence of the ED probability of $\mathrm{Pb}$ nuclei due to collisions with Si nuclei on the momentum $p_{z}$ for the idealized case of the particle motion along the center of the (110) silicon channel when the impact parameter to the channel walls $x_{o}=d_{p} / 2=0.96 \AA$. (b) The dependence of the averaged ED probability of $\mathrm{Pb}$ nuclei with $p_{z}=7 \mathrm{TeV} / c$ on the impact parameter $x_{o}$ to the (110) planes of a silicon crystal in the case of parallel incidence. The dashed line shows the ED probability value averaged by the ensemble of well channeled particles.

value averaged by the particle ensemble $\left(x_{o}\right)$ for a parallel beam, $P_{\text {ed }}=3 \times 10^{-5}$ per $\mathrm{mm}$, is shown by the dashed line.

In a bent crystal, the effective planar potential transforms and the particle trajectory shifts to the outer channel wall. Figure 2 shows the effective potential for $\mathrm{Pb}$ nuclei with $p_{z}=7 \mathrm{TeV} / c$ in the (110) silicon channel bent with the radius $R=100 \mathrm{~m}$ (dot-dashed curve). The well channeled fraction shown by the dashed line is reduced. Besides, the contribution to the $\mathrm{ED}$ of $\mathrm{Pb}$ nuclei from the interactions with the $\mathrm{Si}$ nuclei of the inner channel wall is reduced. However, the ED probability 
averaged by the ensemble of well channeled particles remains close to the value for the straight crystal, $P_{\text {ed }}=$ $2.8 \times 10^{-5}$ per $\mathrm{mm}$.

\section{CONCLUSIONS}

The channeling parameters depend on the ratio of particle momentum to its charge $p_{z}$; therefore the efficiency of the crystal deflector is the same for protons and ions with equal $p_{z}$. The difference appears mainly in multiturn processes of the beam extraction (collimation) because of a much larger cross section for nuclear interactions and larger ionization losses of heavy ions in a crystal, which reduce the contribution of multiple passages of particles through the crystal to the channeling efficiency. Nevertheless, the analysis of the recent collimation experiment with $\mathrm{Pb}$ nuclei of $270 \mathrm{GeV} / c$ per charge at the CERN SPS shows that the channeling efficiency was as large as about $90 \%$.

For $\mathrm{Pb}$ ions of the LHC energies a new mechanism, which can affect the channeling efficiency, appears. The electromagnetic dissociation becomes possible for well channeled particles. However, the estimations performed in the paper show that the ED probability for $\mathrm{Pb}$ nuclei with $p_{z}=7 \mathrm{TeV} / c$ in the (110) silicon channel is sufficiently small, $P_{\text {ed }} \approx 3 \times 10^{-5}$ per $\mathrm{mm}$. For the LHC beam collimation the silicon crystals with the length of 3-5 $\mathrm{mm}$ are planned to be used. Thus, the losses of the well channeled fraction due to ED can be of the order of $0.01 \%$, which should not visibly reduce the collimation efficiency.

On the other hand, the situation realized in the aligned crystal when nuclear interactions are excluded for well channeled nuclei can be favorable for the ED investigation [22]. In particular, the ED probability reduction due to the screening of target nuclei by atomic electrons [24] should be possible to check using channeled $\mathrm{Pb}$ ions of the LHC energies. For realization of such experiments, the selection of well channeled nuclei in the aligned silicon crystal can be performed through the measurement of their ionization losses by the intrinsic semiconductor detector. Besides, the usage of a three-point bending device may give the possibility to practically exclude particles with large oscillation amplitudes in the planar channels for the second half of the crystal.

\section{ACKNOWLEDGMENTS}

We would like to thank Professor G. Smirnov for the photodissociation cross-section data from his collection and useful discussions.

[1] J. Lindhard, K. Dan. Vidensk. Selsk. Mat. Fys. Medd. 34, 14 (1965).

[2] E. N. Tsyganov, Fermilab Reports No. TM-682 and No. TM-684, 1976.

[3] A. S. Vodop'yanov et al., JETP Lett. 30, 442 (1979).

[4] V. V. Biryukov, Yu. A. Chesnokov, and V. I. Kotov, Crystal Channeling and its Application at High-Energy Accelerators (Springer, Berlin, 1997).

[5] A. M. Taratin, Phys. Part. Nucl. 29, 437 (1998).

[6] A. Baurichter et al., Nucl. Instrum. Methods Phys. Res., Sect. B 164-165, 27 (2000).

[7] A. G. Afonin et al., Nucl. Instrum. Methods Phys. Res., Sect. B 234, 14 (2005).

[8] W. Scandale et al., Phys. Lett. B 692, 78 (2010).

[9] W. Scandale et al., Phys. Lett. B 703, 547 (2011).

[10] W. Scandale et al., Phys. Lett. B 714, 231 (2012).

[11] L. I. Bel'zer et al., JETP Lett. 46, 381 (1988).

[12] A. D. Kovalenko, A. M. Taratin, and E. N. Tsyganov, JINR Report No. E1-92-8, 1992.

[13] A. D. Kovalenko, V. A. Mikhailov, A. M. Taratin, and E. N. Tsyganov, JINR Rapid Commun. 6, 13 (1993).

[14] A. D. Kovalenko, V. A. Mikhailov, A. M. Taratin, V. V. Boiko, S.I. Kozlov, and E. N. Tsyganov, JINR Rapid Commun. 4, 9 (1995).

[15] A. S. Artemov et al., JINR Rapid Commun. 2, 46 (1999).

[16] S. V. Afanasiev et al., Part. Nucl. Lett. 1, 42 (2004).

[17] G. Arduini et al., Phys. Rev. Lett. 79, 4182 (1997).

[18] C. Biino et al., Nucl. Instrum. Methods Phys. Res., Sect. B 160, 536 (2000).

[19] C. Biino et al., Nucl. Instrum. Methods Phys. Res., Sect. B 194, 417 (2002).

[20] U. I. Uggerhoj et al., Phys. Lett. B 619, 240 (2005).

[21] R.P. Fliller, A. Drees, D. Gassner, L. Hammons, G. McIntyre, S. Peggs, D. Trbojevic, V. Biryukov, Y. Chesnokov, and V. Terekhov, Nucl. Instrum. Methods Phys. Res., Sect. B 234, 47 (2005).

[22] Yu. L. Pivovarov and V. A. Dolgikh, in Proceedings SPIE 6634, International Conference on Charged and Neutral Particles Channeling Phenomena II, 66340V (2007) [http://dx.doi.org/10.1117/12.741913].

[23] C. A. Bertulani and G. Baur, Phys. Rep. 163, 299 (1988).

[24] Yu. L. Pivovarov, Izv. Vyssh. Uchebn. Zaved., Fiz. 4, 65 (1998). 